\title{
Binge Eating, Body Mass Index, and Gastrointestinal Symptoms
}

\author{
Christine M. Peat, $\mathrm{PhD}^{1,{ }^{*}}$, Lu Huang, $\mathrm{MS}^{2}$, Laura M. Thornton, $\mathrm{PhD}^{1}$, Ann F. Von Holle, MS ${ }^{1}$, \\ Sara E. Trace, $\mathrm{PhD}^{1}$, Paul Lichtenstein, $\mathrm{PhD}^{4}$, Nancy L. Pedersen, $\mathrm{PhD}^{3,4}$, D. Wayne \\ Overby, MD ${ }^{5}$, and Cynthia M. Bulik, PhD $^{1,6}$ \\ ${ }^{1}$ Department of Psychiatry, University of North Carolina, Chapel Hill, North Carolina, USA \\ 2Department of Biostatistics, St. Jude Children's Research Hospital, Memphis, Tennessee, USA \\ ${ }^{3}$ Department of Medical Epidemiology and Biostatistics, Karolinska Institutet, Stockholm, Sweden \\ ${ }^{4}$ Department of Psychology, University of Southern California, Los Angeles, California, USA \\ ${ }^{5}$ Department of Surgery, University of North Carolina, Chapel Hill, North Carolina, USA \\ ${ }^{6}$ Department of Nutrition, University of North Carolina, Chapel Hill, North Carolina, USA
}

\section{Abstract}

Objective-Symptoms of both gastroesophageal reflux disease (GERD) and irritable bowel syndrome (IBS) are frequently reported by individuals who binge eat. Higher body mass index (BMI) has also been associated with these disorders and with binge eating (BE). However, it is unknown whether BE influences GERD/IBS and how BMI might affect these associations. Thus, we examined the potential associations among BE, GERD, IBS, and BMI.

Methods-Participants were from the Swedish Twin study of Adults: Genes and Environment (STAGE) and provided information on disordered eating behavior, BMI, gastrointestinal (GI) disorders, and commonly comorbid psychiatric and somatic illnesses. Key features of GERD and IBS were identified to create modified definitions of both disorders that were used as primary outcome variables. Logistic regression models were applied to determine the association between $\mathrm{BE}$ and each GERD/IBS both independently and in the context of BMI and other commonly comorbid psychiatric and somatic morbidities.

Results-Prevalence estimates for GERD and IBS were higher among women than men (all $p$ values < .001). Only the association between BE and IBS was significant in both men and women after adjustment for BMI and the psychiatric/somatic morbidities.

Conclusion-BE appears to be an important consideration in the presence of IBS symptoms in both men and women, even when considering the impact of BMI and other commonly comorbid

\footnotetext{
(C) 2013 Elsevier Inc. All rights reserved.

"Correspondence to: Cynthia Bulik, PhD, Department of Psychiatry, University of North Carolina at Chapel Hill, CB \#7160, 101 Manning Drive, Chapel Hill, NC 27599-7160, USA. Voice: (919) 843-1689; Fax: (919) 843-8802; cbulik@ med.unc.edu.

Competing interests - None

Conflict of Interest Statement

All authors have completed the Unified Competing Interest form at http://www.icmje.org/coi_disclosure.pdf and they declare that there are no competing interests to report.

All authors reported no biomedical financial interests or potential conflicts of interest.

Publisher's Disclaimer: This is a PDF file of an unedited manuscript that has been accepted for publication. As a service to our customers we are providing this early version of the manuscript. The manuscript will undergo copyediting, typesetting, and review of the resulting proof before it is published in its final citable form. Please note that during the production process errors may be discovered which could affect the content, and all legal disclaimers that apply to the journal pertain.
} 
conditions. This association underscores the importance of routine assessment of BE in patients presenting with IBS to effectively manage the concurrent presentation of these problems.

\section{Keywords}

Binge eating; body mass index; gastroesophageal reflux disease; irritable bowel syndrome

\section{INTRODUCTION}

The association between the behavior of binge eating (BE) and gastrointestinal (GI) complaints is not well understood. BE is the hallmark feature of two eating disordersbulimia nervosa $(\mathrm{BN})$ and binge eating disorder $(\mathrm{BED})$. BN, characterized by recurrent binge eating and compensatory behaviors (e.g., self-induced vomiting, laxative abuse) has been associated with several GI symptoms including acid regurgitation, upper abdominal pain, bloating, and constipation or diarrhea.(1,2) It is not clear whether the observed GI symptoms result from these compensatory behaviors or from the increased volume or composition of food consumed during BE. Further, eating disorder patients with a history of BE commonly report GI symptoms such as impairment of esophageal motility, delayed gastric emptying, bloating, and constipation [1,2].

Two GI disorders frequently reported by individuals with eating disorders (particularly BN and BED) are gastroesophageal reflux disease (GERD) and irritable bowel syndrome (IBS) $[3,4]$. Preliminary studies suggest that individuals who engage in BE are more likely to report upper and lower GI symptoms than individuals who do not have episodes of BE [5]. Additionally, the increased stomach capacity among those who report BE [6] may also negatively impact GI system burden.

The association between $\mathrm{BE}$ and GI symptoms is further complicated by increased body mass index (BMI) in both individuals with BE [7] and in those with a GI disorder [5, 8]. BE [9], GERD [10], and IBS [11] are all frequently reported in obese individuals. Given the associations of BE with both obesity and GI disorders, it is plausible that BE could influence the relationship between BMI and GI disorders.

Thus, the objective of the present study was to investigate the association between BE and GI symptoms, after controlling for BMI. Based on previous studies, we hypothesized that BE would be positively associated with the GERD and IBS even when controlling BMI.

\section{METHOD}

\section{Participants}

Participants were from the Swedish Twin study of Adults: Genes and Environment (STAGE; http://ki.se/ki/jsp/polopoly.jsp?d=9610\&l=en), a large population based study. STAGE is a subset of the Swedish Twin Registry (STR; http://ki.se/twinreg) and includes data collected in 2005 from over 25,000 male and female twins between the ages of 20 and 47 years at time of interview (overall response rate $=59.6 \%$ ). Using web-based questionnaires with a computer assisted phone option, participants provided information on a variety of health related and sociodemographic measures as well as habits and behaviors: $43.1 \%$ responded using the web-based format exclusively and $16.5 \%$ responded exclusively to telephone format. The eating disorders section demonstrated adequate agreement between the web-based questionnaire and the telephone interviews $(K=0.76)$. A detailed description of the study design can be found elsewhere [12,13]. 
A total of 23,821 participants provided information in the eating disorder section. Of these, 23,202 reported information on age at interview, lifetime highest BMI (height and weight), lifetime history of BE (absent or present), and information on GERD or IBS. To be included in the current study, participants must have provided information on purging, anxiety, depression, hypertension, lipid disorders, diabetes, Crohn's disease, and ulcerative colitis (all defined below); participants missing information for any of these variables were excluded from analyses $(n=7,934)$. The resulting sample size available for analyses was 6,827 men and 8,841 women. STAGE was approved by the Regional Ethics Committee at Karolinska Institutet and is in accord with the Declaration of Helsinki. The current study was approved by the Biomedical Institutional Review Board at the University of North Carolina at Chapel Hill. All participants provided informed consent prior to participation.

\section{Disordered Eating Behaviors}

Lifetime history of BE and purging was determined using an expanded Structured Clinical Interview for the Diagnostic and Statistical Manual, Fourth Edition (SCID)-based assessment [14]. For the purposes of this study, BE was assessed using the following two questions: "Have you ever had eating binges when you ate what most people would regard as an unusually large amount of food in a short period of time?" ('yes,' 'no,' and 'do not know/refuse') and "When you were having eating binges, did you feel that your eating was out of control?" ('not at all,' 'slightly,' 'somewhat,' 'very much,' 'extremely,' 'do not know/ refuse'). BE was scored as present if respondents answered 'yes' to the first question and any loss of control to the second question. If respondents answered 'no' to the first question or 'not at all' to the second question, BE was scored as absent.

Purging was defined as engaging in self-induced vomiting and or laxative abuse. Participants were asked, "Which of the following have you ever used to control your shape or weight?' Response options were 'never,' 'once or twice,' 'every week,' or 'daily.' Those who responded 'every week' or 'daily' to either self-induced vomiting or laxative use were scored as positive for purging. In addition, if a participant indicated that $\mathrm{s} / \mathrm{he}$ had eating binges as defined above, s/he was asked if s/he engaged in self-induced vomiting or laxative use during the time of binge eating. Those who responded 'yes' for either self-induced vomiting or laxative use were scored as positive for purging.

\section{BMI Category}

BMI $\left(\mathrm{kg} / \mathrm{m}^{2}\right)$ was calculated using self-reported height and highest self-reported weight (i.e., lifetime highest BMI). For the purposes of this study, BMI was categorized according to World Health Organization guidelines as follows: Underweight $<18.50$; Normal weight $=$ 18.50-24.99; Overweight $=25.00-29.99$; Obese 230.00 [15].

\section{GI Problems}

The primary outcome variables for the current study include symptoms of GERD as determined by the Montreal definition and classification [16] and IBS as determined by the Rome criteria [17]. Participants were asked a series of questions evaluating the presence of GERD and IBS symptoms including its presence (e.g., "Have you ever had Symptom X") and the frequency of that symptom (e.g., "How often do you have Symptom X"). Two definitions of GERD were used in this study: a broad definition and a more specific definition that included an assessment of sleep disturbance. If a respondent reported frequent (> once per month) pain behind the sternum or frequent acid reflux s/he was scored as positive for "GERD broad." For "GERD sleep," if a respondent reported frequent pain behind the sternum or frequent acid reflux and also reported awakening at night due to pain or discomfort behind the sternum, s/he was scored as positive. Similarly, IBS was evaluated using three definitions: 1) recurrent abdominal pain alleviated after defecation (IBS broad); 
2) recurrent abdominal pain occurring at least seven days per month and in the last year that is alleviated after defecation (IBS narrow); and 3) meeting criteria for IBS broad and endorsing either feeling bloated, having constipation, diarrhea, or an incomplete feeling of defecation (IBS cumulative).

\section{Covariate Selection}

Age at interview was included as a covariate in all models because there is an age-related association between BMI and GERD and IBS symptoms [18, 19]. Purging was also included as a covariate in all models in an effort to control for any effects of self-induced vomiting or laxative abuse. In addition, selected psychiatric and medical disorders were considered for inclusion as covariates in the models. Given the demonstrated association between anxiety and depression and both GERD [20] and IBS [21], both psychiatric disorders were evaluated as covariates. Generalized anxiety disorder was considered present for all individuals who reported both DSM-IV-TR Criterion A (excessive anxiety and worry) and Criterion C (at least three symptoms resulting from anxiety and worry). Depression was similarly defined: individuals who reported both DSM-IV-TR Criterion A (five symptoms of depression associated with a change in functioning) and Criterion $\mathrm{C}$ (significant impairment caused by the symptoms) were scored as positive for depression. Given the overlap in some symptom presentations of both ulcerative colitis and Crohn's disease and IBS [22], participants who reported these symptoms were excluded from the analyses $(n=138)$. Additionally, hypertension, lipid disorders, and diabetes have demonstrated associations with BMI [23], therefore these conditions were also evaluated as covariates. To assess these conditions in the current study, participants were asked, "Do you have or have you ever had any of the following problems?" and were instructed to respond 'yes' or 'no' to each disorder in a list which included Crohn's disease, ulcerative colitis, hypertension, lipid disorders, and diabetes.

Anxiety, depression, hypertension, lipid disorder, and diabetes were first evaluated individually for their association with each GI symptom cluster (e.g., GERD broad, GERD sleep, IBS broad, IBS narrow, IBS cumulative) using logistic regression models with generalized estimating equations without correcting for multiple comparisons. Those that were significantly associated with each GI symptom cluster were included in the analytic models described below.

\section{Statistical Analysis}

SAS software (version 9.2) from SAS institute Inc., 2008 [24] was used to perform data analysis. Percent frequencies and means with respective standard deviations were calculated for sample descriptive statistics. Sex differences in the GI outcomes and binge eating were evaluated with chi-square statistics. To evaluate the association of BE with each GI symptom cluster, BE was entered into a logistic regression model as a single predictor. A binge eating $x$ sex interaction was also evaluated in the unadjusted initial models. The interaction was significant in a majority of the models, thus the association between binge eating and each of the GI symptom clusters was evaluated separately by sex. In the second model, anxiety, depression, hypertension, lipid disorders, and diabetes were added as covariates. Lastly, BMI was added in as a covariate in the final model. Generalized estimating equations were applied to account for the non-independence of the data due to inclusion of twins within the same families. To control type I error inflation, p-values were corrected for multiple comparisons using the methods of false discovery rate and families of tests were separated by sex [25]. All corrected $\mathrm{p}$-values $<.05$ were considered statistically significant. 


\section{RESULTS}

\section{Prevalence of GI symptom clusters, covariates, and BMI categories}

In the current sample, the prevalence estimates were: GERD broad $(\operatorname{men}=15.7 \%$; women $=$ $\left.28.9 \% ; X^{2}=352.12, \mathrm{df}=1, \mathrm{p}<.001\right)$, GERD sleep $\left(\right.$ men $=5.5 \%$; women $=12.3 \% ; X^{2}=$ 207.65, $\mathrm{df}=1, \mathrm{p}<.001)$, IBS broad $\left(\operatorname{men}=3.7 \%\right.$; women $=8.1 \% ; X^{2}=115.06, \mathrm{df}=1, \mathrm{p}<$. 001), IBS narrow (men $=1.7 \%$; women $\left.=4.2 \% ; X^{2}=78.78, \mathrm{df}=1, \mathrm{p}<.001\right)$ and IBS cumulative $\left(\right.$ men $=3.1 \%$; women $\left.=7.6 \% ; X^{2}=135.13, \mathrm{df}=1, \mathrm{p}<.001\right)$. Due to these significant sex differences, all analyses were conducted separately by sex. Tables 1 and 2 provide descriptive information regarding the prevalence of each of the GI symptom clusters, the associated covariates, and BMI categories.

\section{The association of BE, medical and psychiatric covariates, and BMI category with GI symptom clusters}

In men, there was a significant positive association between BE and each of the GI symptom clusters (see Figure 1). However, as the covariates were introduced into the model, the associations between BE and GERD sleep (OR $=3.33$, fdr $-p<.05)$, IBS broad (OR $=3.83$, fdr $-p<.05)$, IBS narrow (OR $=6.23$, fdr $-p<.01)$, and IBS cumulative (OR $=4.54$, fdr- $p<$. $01)$ remained significant. When BMI was introduced into the model only the IBS symptom clusters remained significant (IBS broad $\mathrm{OR}=3.84$, fdr- $p<.05$, IBS cumulative $\mathrm{OR}=4.61$, fdr $-p<.01$, and IBS narrow $\mathrm{OR}=6.30$, fdr $-p<.01$ ).

There was a similar pattern of results for women in the current sample (see Figure 1). The initial main effect of BE was significant across all GI symptom clusters (all fdr- $p$ values $<$. 001 ), even in the context of the identified covariates (all fdr- $p$ values $<.05$ ). However, when BMI was introduced into the model, only IBS broad (OR $=1.88$, fdr $-p<.01)$, IBS cumulative $(\mathrm{OR}=1.94$, fdr $-p<.01)$ and IBS narrow $(\mathrm{OR}=1.84$, fdr $-p<.05)$ remained significant.

\section{DISCUSSION}

Prevalence estimates of GERD and IBS symptom clusters in the current study ranged are somewhat lower than those reported in previous studies in the Western world (in both men and women) [26, 27]. The dissimilarities in these prevalence estimates may be suggestive of true differences among individuals reporting GI symptoms in Sweden; however, it is possible that the lower prevalence was influenced by an overall lower BMI among participants in this cohort. The majority of individuals in the current sample fell within a normal BMI range $\left(18.50-24.99 \mathrm{~kg} / \mathrm{m}^{2}\right)$ and given the demonstrated effect of weight on both GERD [10] and IBS [11], it is possible that the lower prevalence of the GI symptoms in this study reflected this fact. Alternatively, it is possible that the lower prevalence of GERD in our study is reflective of a difference among Swedish men as one recent study failed to find any significant association between BMI and GERD symptoms in this group [28]. Despite these differences, GERD and IBS were reported among a sizeable proportion of individuals in the sample, and this was particularly true for reports of GERD among women.

The significant, positive association between BE and IBS symptoms, which persisted even after introducing potential medical and psychiatric morbidities (e.g., hypertension, Crohn's disease, depression) and BMI, suggests that the influence of BE is particularly robust. Given the exploratory nature of the current analyses we present three potential interpretations of the underlying association between BE and IBS. First, both BE and IBS have demonstrated important relationships with psychosocial stress [29-31]. Stress is known to be a common precipitant of BE [29] and many individuals who struggle with this behavior may be prone to using BE in an effort to reduce anxiety or dysphoria [31]. In addition, stress is thought to 
play an important etiological role in functional GI disorders (the most common of which is IBS) [30, 32-35]. The observed association could potentially reflect a pattern that would be expected to occur if stress were a critical third variable that influenced both BE and IBS. While the current analyses do not lend themselves to mediation to assess the influence of stress, such analyses would be important to undertake in future studies.

A second hypothesis is that the acute symptoms of IBS (e.g., nausea, diarrhea) may cause patients to either severely restrict their diets so as to avoid specific foods or to undergo prolonged periods of restriction to avoid IBS flares. Given the known association between dietary restriction and urges to binge [36], the observed association might suggest an inadvertent side effect of attempts to avoid IBS episodes via food restriction or limitation.

A third hypothesis is that individuals with concurrent BE and IBS may experience acute symptoms of IBS as a result of bingeing on foods that are not well tolerated. Symptoms of IBS are often triggered by food intolerance, particularly with foods that are high in fat content [37-39]. Macronutrient studies of those who engage in BE reveal that typical binge episodes are dense with high-fat foods and carbohydrates [40]. Thus, patients who struggle with BE may be more prone to IBS flare ups given that their binges are likely to be rich with foods that are poorly tolerated due to IBS. The design of the current study precluded testing of these alternative explanations for our observation; however, it will be important for future studies to examine the role of stress, food avoidance/fasting, and macronutrient content in the association between BE and IBS. Examination of the temporal sequencing of IBS and $\mathrm{BE}$ onset will be particularly relevant given our limited understanding of the association between these variables.

Thus, the current pattern of results underscores the importance of the regular evaluation of $\mathrm{BE}$ in the context of IBS. Not only was BE reported among a sizeable portion of the sample, BE was more likely in those reporting both GERD and IBS even when purging was controlled statistically. Given this significant finding, it is prudent to consider screening for $\mathrm{BE}$ as a regular part of GERD and IBS evaluations, especially among women. This is particularly salient as recent estimates have suggested that BED is the most common of all eating disorder pathology with estimates as high as 3.5 percent among women and 2.0 percent among men in the United States [41].

The current findings also have important implications for the management of patients who are reporting concurrent BE and GI symptoms. Interventions for IBS should not only focus on the alleviation of symptoms like abdominal pain and diarrhea, but also on the elimination of any concurrent BE. It stands to reason that depending on the causal relationships between $\mathrm{BE}$ and IBS, it is possible that as BE improves, a reduction in IBS symptoms and/or severity might occur. A combined medical and behavioral treatment plan would require a multidisciplinary approach common to many GI practices throughout the world given the frequently associated psychological factors that can exacerbate many GI disorders [42]. In the case of $\mathrm{BE}$, however, it would be sensible to ensure that patients are receiving psychological care from eating disorder specialists who are trained in the management of eating pathology. In fact, regular screening of eating pathology in GI clinics could help facilitate an appropriate referral and perhaps expedite the treatment process for patients struggling with concurrent BE and GI symptoms.

\section{Limitations}

Although the current study provides important insights regarding the role of $\mathrm{BE}$ in the relationship between BMI and GI symptoms, there are a number of notable limitations. First, the population surveyed in the present sample was Swedish men and women thus the results might not generalize to other ancestry groups. Second, although the current data were 
derived from a large, population-based register, it is possible that the results are not entirely representative of the population given that the initial response rate was 59.6\%. Third, the report of loss of control with regard to BE was rarely reported among Swedish men. This lack of reporting might reflect the true experience of Swedish men; however it is also possible that the construct was not defined in a way that resonated with the population being sampled. Thus our estimates of BE among men are likely to be biased given the relatively infrequent report of loss of control eating. Fourth, the data analyzed in this study were crosssectional and the time span corresponding to reported symptoms is not consistent across measures. For example, BE, anxiety, depression, and BMI were assessed over the lifetime, whereas GI symptoms (including those consistent with GERD and IBS) reflected an individual's current status. It is therefore important to recognize that the associations elucidated here are correlational in nature and causal inference cannot be drawn. The time span discrepancies in the data collection also necessitate caution when interpreting the correlational findings. This limitation highlights the importance of longitudinal research designs in determining the nature of the associations between BE, BMI, and GI symptoms. Fourth, the GI symptom clusters used for the current study do not reflect full diagnoses of GERD or IBS. Although the symptom criteria used to describe each cluster were drawn from established definitions and diagnostic guidelines, it is possible that the selection of other criteria might have resulted in a different outcome. Future studies would benefit from assessing full diagnostic criteria for both GERD and IBS to further evaluate the potential impact of BE on these diagnoses. Finally, the data collected for the current study did not differentiate between Type 1 or Type 2 diabetes and thus we are unable to determine the extent to which either presentation independently might have influenced the results. It will be important for future studies to assess specific diabetes diagnoses and how each might affect the association between BE and GI disorders.

The results of the current study provide empirical support for an association between BE and GI symptoms consistent with IBS in both men and women. This study is novel in its approach in that we were able to assess the independent effect of BE while controlling for any potential impact that purging might introduce. Thus the results shed new light on a previously understudied phenomenon and provide direction for future research and clinical interventions. Empirical investigations that apply longitudinal designs and integrate full diagnoses of GERD and IBS are necessary to understand the impact of BE more fully. It will also be necessary to evaluate the nature of these associations in men and women of other racial and ethnic backgrounds given that GI symptoms consistent with these disorders have been shown to disproportionately affect individuals of African American and Hispanic descent [43-45]. The current findings suggest that clinical practice might also benefit from a more regular screening of BE when evaluating GERD and IBS. Additionally, management of any relevant $\mathrm{BE}$ should be incorporated in designing and tailoring treatment interventions given that the continued presence of this behavior may exacerbate symptoms or prevent optimum treatment outcomes.

\section{Acknowledgments}

We would like to thank all participants for their time and effort.

Funding: Drs. Peat and Trace were supported by the National Institute of Mental Health grant T32MH076694 (PI: Bulik). The Swedish Twin Registry is supported by grants from the Swedish Department of Higher Education, the Swedish Research Council. We thank all participants for their time and efforts. 


\section{References}

1. Boyd C, Abraham S, Kellow J. Psychological features are important predictors of functional gastrointestinal disorders in patients with eating disorders. Scand J Gastroenterol. 2005; 40:929-35. [PubMed: 16170899]

2. Zipfel S, Sammet I, Rapps N. Gastrointestinal disturbances in eating disorders: clinical and neurobiological aspects. Auton Neurosci. 2006; 129:99-106. [PubMed: 16962383]

3. Crowell M, Cheskin L, Musial F. Prevalence of gastrointestinal symptoms in obese and normal weight binge eaters. Am J Gasteroenterol. 1994; 89:387.

4. Georg S. Gut function in anorexia nervosa and bulimia nervosa. Scand J Gastroenterol. 2003; 38:573-87. [PubMed: 12825864]

5. Cremonini F, Camilleri M, Clark M, Beebe T, Locke G, Zinsmeister A, et al. Associations among binge eating behavior patterns and gastrointestinal symptoms: a population-based study. Int J Obes. 2009; 33:342-53.

6. Geliebter A, Yahav EK, Gluck ME, Hashim SA. Gastric capacity, test meal intake, and appetitive hormones in binge eating disorder. Physiol Behav. 2004 Jul.81:735-40. [PubMed: 15234178]

7. Grucza RA, Przybeck TR, Cloninger CR. Prevalence and correlates of binge eating disorder in a community sample. Compr Psychiatry. 2007; 48:124-31. [PubMed: 17292702]

8. Delgado-Aros S, Locke GR, Camilleri M, Talley NJ, Fett S, Zinsmeister AR, et al. Obesity is associated with increased risk of gastrointestinal symptoms: a population-based study. Am J Gasteroenterol. 2004; 99:1801-6.

9. Stunkard AJ. Eating disorders and obesity. Psychiatr Clin North Am. 2011; 34:765-71. [PubMed: 22098802]

10. Van Oijen M, Josemanders D, Laheij R, Van Rossum L, Tan A, Jansen J. Gastrointestinal disorders and symptoms: does body mass index matter. Neth J Med. 2006; 64:45-9. [PubMed: 16517988]

11. Svedberg P, Johansson S, Wallander MA, Hamelin B, Pedersen N. Extra-intestinal manifestations associated with irritable bowel syndrome: a twin study. Aliment Pharmacol Ther. 2002; 16:97583. [PubMed: 11966507]

12. Furberg H, Lichtenstein P, Pedersen NL, Thornton L, Bulik CM, Lerman C, et al. The STAGE cohort: a prospective study of tobacco use among Swedish twins. Nicotine Tob Res. 2008; 10:1727-35. [PubMed: 18988069]

13. Lichtenstein P, Sullivan PF, Cnattingius S, Gatz M, Johansson S, Carlstrom E, et al. The Swedish Twin Registry in the third millennium: an update. Twin Res Hum Genet. 2006; 9:875-82. [PubMed: 17254424]

14. First, M.; Gibbon, M.; Williams, J.; Spitzer, P.; Staff, M. Computer Program for Windows TM, Software Manual. Washington, DC: American Psychiatric Press, Inc; 1999. SCID Screen Patient Questionnaire (SSPQ) and SCID SCREEN Patient Questionnaire-Extended (SSPQ-X).

15. World Health Organization. Obesity: preventing and managing the global epidemic: report of a WHO consultation. Geneva, Switzerland: World Health Organization; 2000.

16. Vakil N, van Zanten SV, Kahrilas P, Dent J, Jones R. The Montreal definition and classification of gastroesophageal reflux disease: a global evidence-based consensus. Am J Gastroenterol. 2006; 101:1900-20. [PubMed: 16928254]

17. Drossman DA, Dumitrascu DL. Rome III: New standard for functional gastrointestinal disorders. J Gastrointestin Liver Dis. 2006; 15:237. [PubMed: 17013448]

18. Kubo M, Fujiwara Y, Shiba M, Kohata Y, Yamagami H, Tanigawa T, et al. Differences between risk factors among irritable bowel syndrome subtypes in Japanese adults. Neurogastroenterol Motil. 2011; 23:249-54. [PubMed: 21122032]

19. Ruigómez A, García Rodríguez LA, Wallander MA, Johansson S, Graffner H, Dent J. Natural history of gastro-oesophageal reflux disease diagnosed in general practice. Aliment Pharmacol Ther. 2004; 20:751-60. [PubMed: 15379835]

20. Jansson C, Nordenstedt H, Wallander MA, Johansson S, Johnsen R, Hveem K, et al. Severe gastrooesophageal reflux symptoms in relation to anxiety, depression and coping in a population-based study. Aliment Pharmacol Ther. 2007; 26:683-91. [PubMed: 17697202] 
21. Levy RL, Olden KW, Naliboff BD, Bradley LA, Francisconi C, Drossman DA, et al. Psychosocial aspects of the functional gastrointestinal disorders. Gastroenterology. 2006; 130:1447-58. [PubMed: 16678558]

22. Baumgart DC, Sandborn WJ. Inflammatory bowel disease: clinical aspects and established and evolving therapies. Lancet. 2007; 369:1641-57. [PubMed: 17499606]

23. Guh D, Zhang W, Bansback N, Amarsi Z, Birmingham CL, Anis A. The incidence of co morbidities related to obesity and overweight: a systematic review and meta-analysis. BMC Public Health. 2009; 9:88. [PubMed: 19320986]

24. SAS/STAT® 9.2 User's Guide [program]. 9.2 version. Cary, NC: SAS Institute Inc; 2008.

25. Benjamini Y, Hochberg Y. Controlling the false discovery rate: a practical and powerful approach to multiple testing. J R Stat Soc Series B Stat Methodol. 1995; 57:289-300.

26. Dent J. Epidemiology of gastro-oesophageal reflux disease: a systematic review. Gut. 2005; 54:710-7. [PubMed: 15831922]

27. Hungin APS, Chang L, Locke GR, Dennis EH, Barghout V. Irritable bowel syndrome in the United States: prevalence, symptom patterns and impact. Aliment Pharmacol Ther. 2005; 21:1365-75. [PubMed: 15932367]

28. Lagergren J, Bergstrom R, Nyren O. No relation between body mass and gastro oesophageal reflux symptoms in a Swedish population based study. Gut. 2000 Jul.47:26-9. [PubMed: 10861260]

29. Freeman LM, Gil KM. Daily stress, coping, and dietary restraint in binge eating. Int J Eat Disord. 2004 Sep.36:204-12. [PubMed: 15282690]

30. Murray CDR, Flynn J, Ratcliffe L, Jacyna MR, Kamm MA, Emmanuel AV. Effect of acute physical and psychological stress on gut autonomic innervation in irritable bowel syndrome. Gastroenterology. 2004; 127:1695-703. [PubMed: 15578507]

31. Telch CF, Agras WS, Linehan MM. Dialectical behavior therapy for binge eating disorder. J Consult Clin Psychol. 2001; 69:1061-5. [PubMed: 11777110]

32. Almy TP. Experimental studies on the irritable colon. Am J Med. 1951; 10:60-7. [PubMed: 14799500]

33. Kay L, Jorgensen T, Jensen KH. The epidemiology of irritable bowel syndrome in a random population: prevalence, incidence, natural history and risk factors. J Intern Med. 1994; 236:23-30. [PubMed: 8021568]

34. Stam R, Akkermans LM, Wiegant VM. Trauma and the gut: interactions between stressful experience and intestinal function. Gut. 1997; 40:704-9. [PubMed: 9245921]

35. Welgan P, Meshkinpour H, Beeler M. Effect of anger on colon motor and myoelectric activity in irritable bowel syndrome. Gastroenterology. 1988; 94:1150-6. [PubMed: 3350284]

36. Engelberg MJ, Gauvin L, Steiger H. A naturalistic evaluation of the relation between dietary restraint, the urge to binge, and actual binge eating: a clarification. Int J Eat Disord. 2005; 38:35560. [PubMed: 16231355]

37. Dapoigny M, Stockbrugger RW, Azpiroz F, Collins S, Coremans G, Muller-Lissner S, et al. Role of alimentation in irritable bowel syndrome. Digestion. 2003; 67:225-33. [PubMed: 12966230]

38. Floch MH, Narayan R. Diet in the irritable bowel syndrome. J Clin Gastroenterol. 2002; 35:S4552. [PubMed: 12184139]

39. Lea R, Whorwell PJ. The role of food intolerance in irritable bowel syndrome. Gastroenterol Clin North Am. 2005; 34:247-55. [PubMed: 15862933]

40. Bartholome LT, Raymond NC, Lee SS, Peterson CB, Warren CS. Detailed analysis of binges in obese women with binge eating disorder: Comparisons using multiple methods of data collection. Int J Eat Disord. 2006; 39:685-93. [PubMed: 16937383]

41. Hudson J, Hiripi E, Pope HG, Kessler RC. The prevalence and correlates of eating disorders in the national comorbidity survey replication. Biol Psychiatry. 2007; 61:348-58. [PubMed: 16815322]

42. Locke GR, Weaver AL, Melton LJ, Talley NJ. Psychosocial factors are linked to functional gastrointestinal disorders: a population based nested case-control study. Am J Gastroenterol. 2004; 99:350-7. [PubMed: 15046228] 
43. El-Serag HB, Petersen NJ, Carter J, Graham DY, Richardson P, Genta RM, et al. Gastroesophageal reflux among different racial groups in the United States. Gastroenterology. 2004; 126:1692-9. [PubMed: 15188164]

44. Wang A, Mattek NC, Holub JL, Lieberman DA, Eisen GM. Prevalence of complicated gastroesophageal reflux disease and Barrett's esophagus among racial groups in a multi-center consortium. Dig Dis Sci. 2009; 54:964-71. [PubMed: 19255852]

45. Yuen E, Romney M, Toner RW, Cobb NM, Katz PO, Spodik M, et al. Prevalence, knowledge and care patterns for gastro-oesophageal reflux disease in United States minority populations. Aliment Pharmacol Ther. 2010; 32:654-55. 


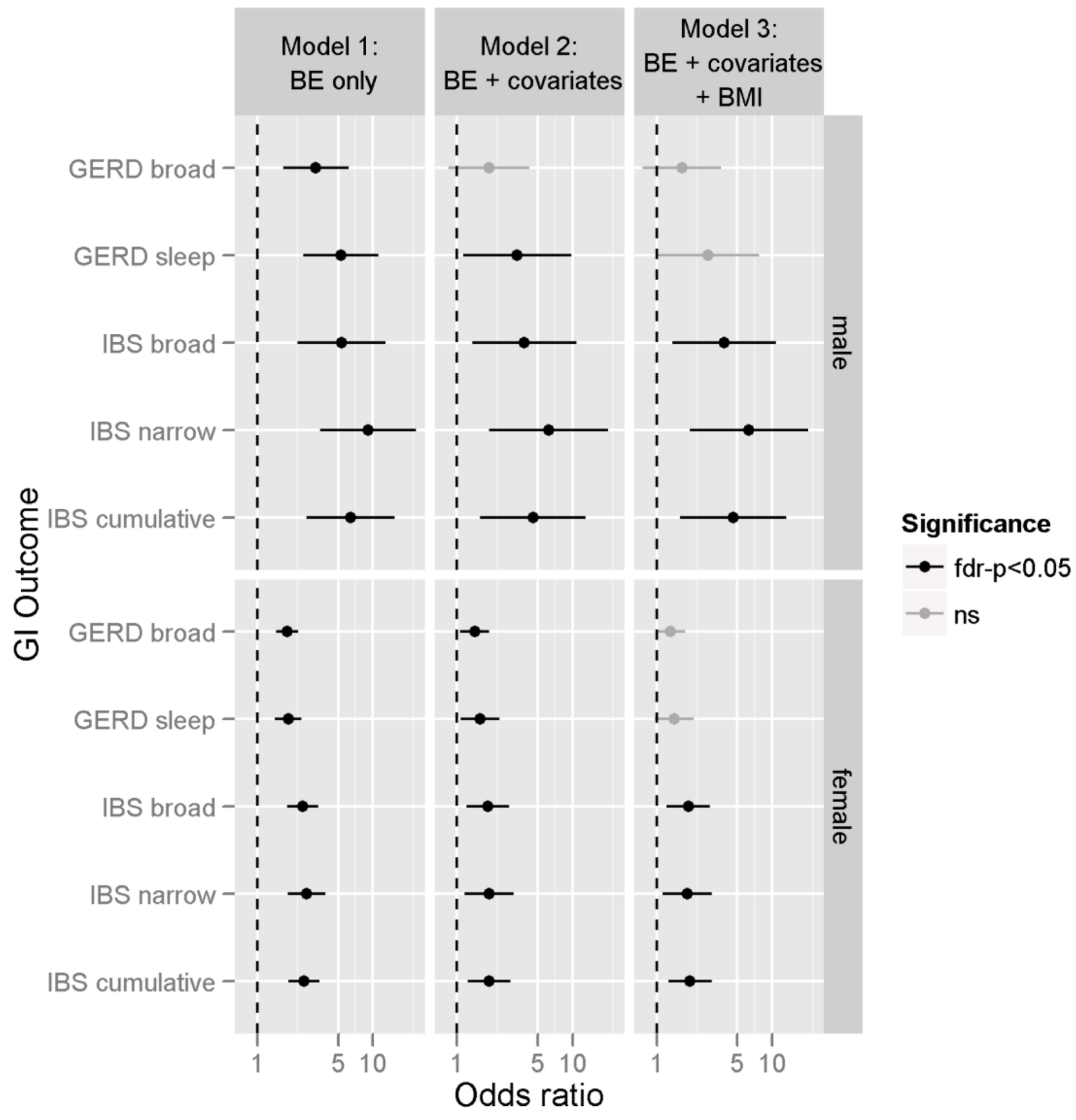

Figure I.

Odds Ratio of Gastrointestinal (GI) Outcomes by Binge Eating (BE) Status.

${ }^{a}$ Note. The dots represent odds ratios and the intervals represent $95 \%$ confidence intervals. 


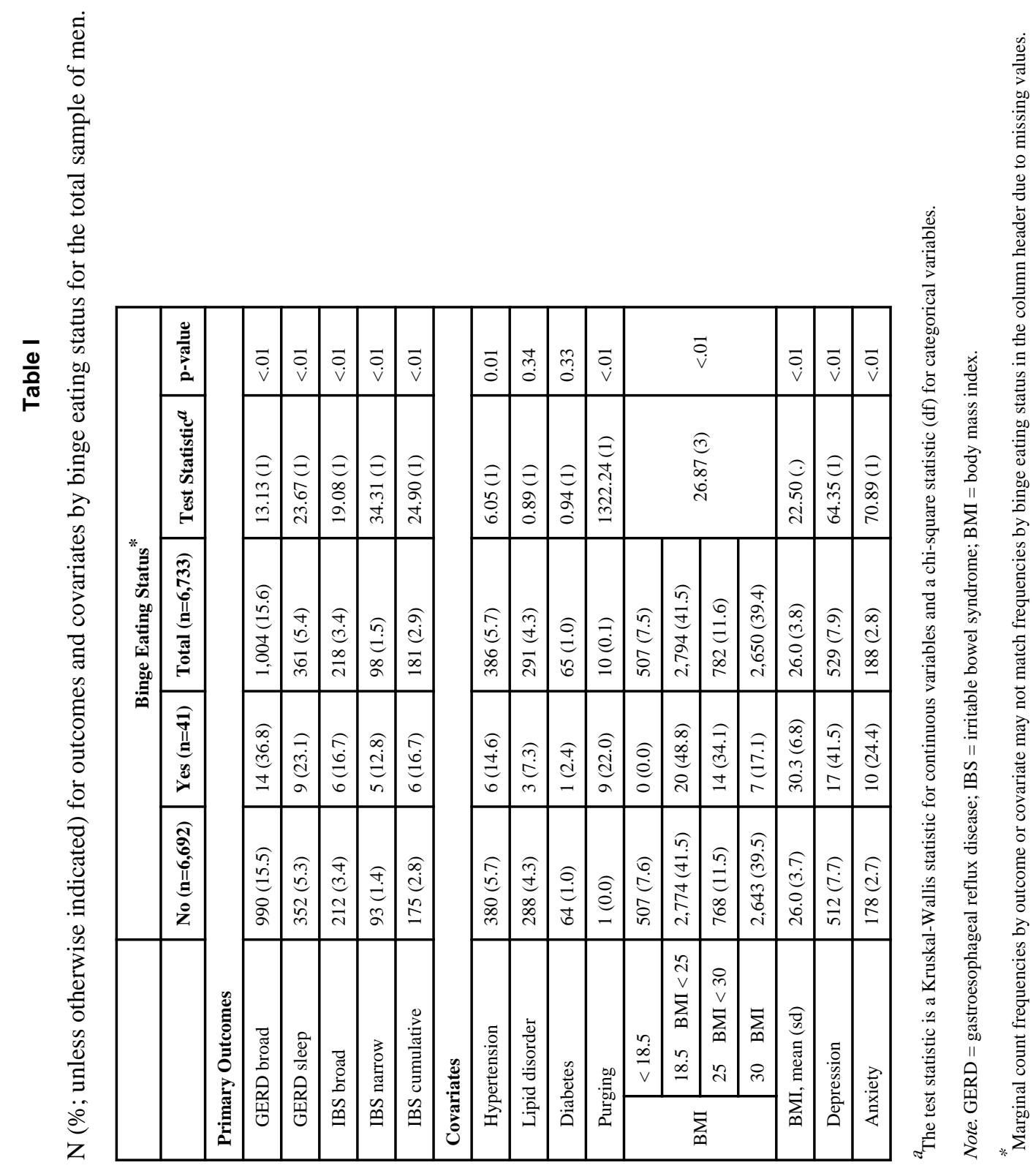


